\title{
BMJ Open Group cognitive remediation therapy for adults with obesity prior to behavioural weight loss treatment: study protocol for a randomised controlled superiority study (CRT study)
}

\author{
Anja Hilbert, ${ }^{1}$ Marie Blume, ${ }^{1}$ David Petroff, ${ }^{1,2}$ Petra Neuhaus, ${ }^{2}$ Evelyn Smith,,${ }^{3,4}$ \\ Phillipa J Hay, ${ }^{4}$ Claudia Hübner ${ }^{1}$
}

To cite: Hilbert A, Blume M, Petroff D, et al. Group cognitive remediation therapy for adults with obesity prior to behavioural weight loss treatment: study protocol for a randomised controlled superiority study (CRT study). BMJ Open 2018;8:e022616. doi:10.1136/ bmjopen-2018-022616

\section{- Prepublication history for} this paper is available online. To view these files, please visit the journal online (http://dx.doi. org/10.1136/bmjopen-2018022616).

Received 26 February 2018 Revised 1 June 2018 Accepted 29 June 2018

D Check for updates

(c) Author(s) (or their employer(s)) 2018. Re-use permitted under CC BY-NC. No commercial re-use. See rights and permissions. Published by BMJ.

For numbered affiliations see end of article.

\section{Correspondence to}

Professor Anja Hilbert; anja.hilbert@medizin.unileipzig.de

\section{ABSTRACT}

Introduction Individuals with obesity show deficits in executive functioning which have been implicated in decreased weight loss outcome. Preliminary evidence suggests that cognitive remediation therapy (CRT) improves executive functioning and weight loss in obesity. However, confirmatory support, especially for pre-weight loss use, is lacking. The CRT study aims at determining the efficacy of CRT versus no treatment control in patients with obesity before entering behavioural weight loss (BWL) treatment. It is hypothesised that individuals who receive CRT will show better weight loss outcome, improved executive functioning, greater weight loss-related behavioural changes and higher attendance of BWL treatment, 6 and 12 months after cessation of CRT.

Methods and analysis In a single-centre, assessorblinded, randomised, two-armed parallel-group superiority trial, 260 adults with body mass index $\geq 35.0 \mathrm{~kg} / \mathrm{m}^{2}$ are centrally randomised to 8 -week group-based CRT versus no treatment, before entering BWL treatment. Primary outcome is the amount of weight loss (\%) at 6-month follow-up, compared with pre-treatment, derived from measured body weight. Secondary outcomes include improvement in executive functioning post-treatment and in weight loss-related behaviour, mental and physical health, and attendance to BWL treatment at 6-month and 12-month follow-up. Maintenance of weight loss at 12-month follow-up will be determined. Mixed model analyses based on intentto-treat will be used to compare the CRT and control groups with respect to differences in weight change between pre-treatment and 6-month follow-up. Similar models will be used for analysing 12-month follow-up data and secondary outcomes. Further analyses will include additional covariates to identify predictors of treatment outcome.

Ethics and dissemination The study was approved by the Ethical Committee of the University of Leipzig (25615-13072015, version 'Final 1.0 from 28 May 2015). The study results will be disseminated through peerreviewed publications.

Trial registration number DRKS00009333; Pre-results.

\section{Strengths and limitations of this study}

- This is the first study to evaluate the short-term and long-term efficacy of cognitive remediation therapy versus no treatment control condition in adults with obesity before entering behavioural weight loss treatment in a prospective, randomised-controlled trial.

- Novel features include the comprehensive therapeutic focus on executive functioning deficits in obesity that may be assumed to underlie weight loss failure, the use of computerised training exercises and adaptation to group format.

- The study is well-controlled, adequately powered and uses established outcome measures.

- Limitations include being single-centred and deploying a small number of therapists.

\section{INTRODUCTION}

Prevalence rates for obesity and overweight in adults have increased over the prior decades. ${ }^{12}$ Obesity is a leading cause of health-related disorders, such as type 2 diabetes mellitus and cardiovascular disease and premature mortality. ${ }^{34}$ This sequelae can be alleviated through modest weight loss ${ }^{5-7}$ that can be achieved with behavioural weight loss (BWL) programmes, ${ }^{8}$ the standard in obesity treatment. ${ }^{9}$ However, in the long term, many BWL patients regain the majority of weight initially reduced. ${ }^{10}$ Recently, weight loss failure has been linked to detriments in cognitive function, especially in executive function, which encompasses a range of higher cognitive capacities that enable forethought and the regulation of complex goal-oriented behaviour ${ }^{11}{ }^{12}$-and thus self-regulation. ${ }^{13}$ In general, individuals with obesity present with impairment in executive functions. ${ }^{14-16}$ Indeed, in their meta-analysis, 
Yang et al confirmed that inhibition, flexibility, working memory, planning, decision-making and verbal fluency are impaired in individuals with obesity versus those with normal weight, ${ }^{17}$ although moderators such as education or socioeconomic status could not sufficiently be examined.

The mechanisms linking executive function to obesity are complex and not fully understood. Theoretical models conceptualised a bidirectional association. ${ }^{1518}$ On the one hand, executive function is likely to underlie behaviours of successful weight management,${ }^{19-21}$ for example, goal setting, self-monitoring, slowing eating rate and physical activity. ${ }^{22}{ }^{23}$ Indeed, first evidence linking specific executive functions to weight loss-related behaviours in individuals with obesity showed that reduced inhibition and planning abilities longitudinally predicted lower dietary quality, including lower consumption of fruits and vegetables, ${ }^{24}$ extending results from non-obese samples. ${ }^{25-27}$ In addition, executive functions moderated the association between dietary intentions or preferences and later eating behaviour or weight outcome in non-obese samples. ${ }^{28} 29$ Among the few studies that have addressed the predictive value of executive functions for weight loss outcome in obesity, difficulties in executive function, including impaired inhibition, decision-making and flexibility predicted lesser weight loss in individuals with obesity treated with BWL ${ }^{3031}$ and bariatric surgery. ${ }^{32}$ In turn, weight loss itself has been found to be associated with improvements in executive functioning, attention and memory in individuals with obesity. ${ }^{33} 34$

On the other hand, a deleterious effect of obesity on executive functions has been found. ${ }^{15} 18$ Although obesity-related medical sequelae, including the metabolic syndrome and type 2 diabetes mellitus, ${ }^{35} 36$ may contribute, these comorbidities are unlikely to account fully for the association between obesity and executive functioning. ${ }^{17}$ As potential biological pathways explaining the link between obesity and impaired executive functions, obesity-related inflammation, ${ }^{37-39}$ metabolic factors such as hyperglycaemia, hyperinsulinaemia and hypertension that increase the risk for microstructural brain damage,$^{35} 36$ and genetic factors ${ }^{41}$ have been proposed. Functional MRI studies in obesity, mirroring the neuropsychological findings on executive function impairment, ${ }^{17}$ document a differential brain activation in response to food cues in areas related to inhibitory control and reward processing, ${ }^{42}$ suggesting a hypofunctioning of the dorsal control pathway and a hyperfunctioning of the ventral reward pathway. ${ }^{43}$ Initial studies found lower reward-sensitive decision-making and food-related activation of the dorsal control pathway to be longitudinally predictive of weight loss after a dietary intervention and weight loss maintenance, ${ }^{445}$ suggesting a predictive value of executive functions for long-term weight loss outcome.

Despite the plausible relevance of executive functioning for weight management, research has only begun to develop neurocognitive interventions to improve these functions. As systematically reviewed by Jones $e t a l$, increasing experimental evidence suggests that neuropsychological trainings of specific executive functions can improve working memory as well as food-related inhibitory control and attentional bias, ${ }^{46}$ but overall evidence was limited and inconclusive, for example, regarding general inhibitory control. Validation of neuropsychological training effects outside the laboratory and clinical application to individuals with obesity was notably lacking, and the mechanisms of action remained unclear.

A more comprehensive clinical approach to treatment that has recently been applied to obesity is cognitive remediation therapy (CRT). CRT was developed in order to improve basic neurocognitive functions in patients with brain lesions ${ }^{47}$ and schizophrenia ${ }^{48}$ and was successfully adapted to other disorders, for example, depression, ${ }^{49}$ attention deficit/hyperactivity disorder ${ }^{50}$ and anorexia nervosa. ${ }^{51}{ }^{52}$ The first (to our knowledge) open randomised-controlled trial (RCT) in 80 adults with obesity who had undergone a brief group-based BWL intervention found that a post-BWL individual CRT with eight sessions focusing on enhancing cognitive flexibility versus no CRT improved cognitive flexibility at post-treatment and 3-month follow-up and led to a greater weight loss at 3-month follow-up. ${ }^{53}$ The improvement in cognitive flexibility significantly predicted greater weight loss, thus supporting the postulated mechanism of action. A further RCT of individual CRT as a preparatory adjunct to BWL treatment versus BWL treatment only in adults with obesity is under way. ${ }^{54}$

This research suggests that CRT may represent a valuable addition to standard BWL treatment in individuals with obesity. CRT could further increase weight loss outcome when conducted prior to BWL by enhancing patient motivation, for example, self-determination in weight loss-promoting behaviours, ${ }^{55}$ and attendance and retention in BWL, all aspects known to be associated with greater weight loss. ${ }^{2356}$ As obesity and the associated medical comorbidity increase healthcare costs, ${ }^{57} 58$ CRT for obesity, if successful, has the potential to provoke a reduction of these costs. While the previously used CRT protocol for obesity focused mainly on enhancing cognitive flexibility including set-shifting, ${ }^{59}$ a more comprehensive focus on other aspects of impaired executive function in obesity, including inhibition, planning and decision-making, ${ }^{17}$ as well as attentional bias to salient food is promising. ${ }^{60}$ Incorporation of computerised neuropsychological exercises is timely and suited to ensure standardised administration. ${ }^{6162}$ In addition, an adaptation to group format could foster dissemination of CRT following demonstration of its efficacy, because group CRT could easily and with potential cost-effectiveness be added to BWL which is typically provided in group format. ${ }^{63}$

This study, thus, aims at determining the efficacy of CRT versus no treatment control condition in patients with obesity prior to BWL treatment in a single-centre, assessor-blinded, randomised, two-armed parallel-group, superiority study (CRT study). Safety and potential efficacy of the intervention were assumed based on trials 
comparable to the CRT study, in which serious adverse events have not been reported. ${ }^{5153}$ In patients with obesity, one study found CRT to significantly improve executive functioning and weight outcome without increasing psychopathology. ${ }^{53}$

The hypotheses of the CRT study are as follows:

1. Patients receiving CRT will show increased weight loss compared with patients receiving no CRT treatment at 6-month follow-up. (As CRT does no focus on weight loss, but on the executive functions that are assumed to underlie weight loss behaviours, we expect a difference in weight loss between the CRT and the no treatment control condition to occur after 6 months of subsequent BWL.)

2. Patients receiving CRT will show improvements in executive functioning, behavioural indicators of weight loss, mental and physical health, and higher attendance and retention in BWL treatment compared with patients receiving no CRT treatment at post-treatment and at 6-month and 12-month follow-up. (Change in executive functioning to be evaluated at post-treatment only.)

3. Patients receiving CRT will sustain larger amounts of weight loss compared with patients receiving no CRT treatment at 12-month follow-up.

\section{METHODS AND ANALYSIS}

Design, participants and procedures

Study design

The CRT study is a single-centre, assessor-blinded, randomised, two-armed parallel-group superiority study, evaluating the efficacy of CRT (experimental condition) compared with no treatment (control condition) in adults with obesity prior to entering BWL treatment. The study design is depicted in figure 1 .

The study period lasts 14 months per patient. Following enrolment in the BWL treatment programme at the Outpatient Unit of the Integrated Research and Treatment Centre (IFB) AdiposityDiseases and after determining eligibility for the CRT study, patients are randomised to 2 months of CRT versus no treatment. After 2 months, all patients participate in BWL treatment up to 12 months.

\section{Participants}

A total of 260 adult patients with obesity class 2 or 3 (body mass index, BMI $\left.\geq 35.0 \mathrm{~kg} / \mathrm{m}^{2}\right)^{64}$ seeking BWL treatment are randomised to either CRT treatment or no treatment. Inclusion criteria are summarised in box 1 . To ensure generalisation of study results, exclusion criteria are kept to a minimum.

\section{Recruitment}

This ongoing study is being conducted from May 2015 to July 2019 at the Outpatient Unit of the IFB AdiposityDiseases at University of Leipzig Medical Centre, Leipzig, Germany. Recruitment strategies include the presentation of the CRT study at an information session for all patients presenting at the IFB Outpatient Unit for BWL treatment. In addition, patients are recruited from the community via public notices and advertisements (placed in, eg, newspapers, websites, clinics and facilities of the University of Leipzig, primary care physicians, pharmacies, stores and public transport). Patients are offered a

\begin{tabular}{|c|c|c|c|c|c|c|}
\hline \multirow[b]{3}{*}{ Time point } & \multicolumn{6}{|c|}{ Study period } \\
\hline & \multirow{2}{*}{$\begin{array}{c}\text { Screening } \\
-t_{2}\end{array}$} & \multirow{2}{*}{$\begin{array}{c}\text { Enrolment } \\
\qquad t_{1}\end{array}$} & \multirow{2}{*}{$\begin{array}{c}\text { Pre-treatment } \\
\qquad t_{0}\end{array}$} & \multicolumn{3}{|c|}{ Follow-up } \\
\hline & & & & $t_{1}$ & $t_{2}$ & $t_{3}$ \\
\hline \multicolumn{7}{|l|}{ Enrolment } \\
\hline Eligibility & $x$ & $x$ & & & & \\
\hline Informed consent & & $x$ & & & & \\
\hline Allocation & & $x$ & & & & \\
\hline \multicolumn{7}{|l|}{ Interventions } \\
\hline \multicolumn{7}{|l|}{ Cognitive remediation therapy } \\
\hline No treatment & & & $\leftarrow$ & & & \\
\hline \multicolumn{7}{|l|}{ Assessments } \\
\hline $\begin{array}{l}\text { Weight loss, executive functioning, weight loss-related behaviours, mental } \\
\text { and physical health, attendance of behavioural weight loss treatment }\end{array}$ & & & $x$ & $x$ & $x$ & $x$ \\
\hline
\end{tabular}

Figure 1 Schedule of enrolment, interventions and assessments. t1, post-treatment; t2, 6-month follow-up; t3, 12-month follow-up. Executive functioning is assessed at $\mathrm{t} 0$ and $\mathrm{t} 1$ only. 


\section{Box 1 Inclusion and exclusion criteria}

Inclusion
Age $\geq 18$ years.
Body mass index $\geq 35.0 \mathrm{~kg} / \mathrm{m}^{2}$.
Presenting for behavioural weight loss treatment at the IFB
Outpatient Unit.
Feasible participation in the study procedures at the IFB Research
Unit.
Sufficient German language skills.
Informed consent.
Exclusion
Serious somatic conditions (eg, neurological disorders, stroke, head
injury).
Serious mental conditions (eg, psychotic disorder, suicidality, sub-
stance use disorder, attention deficit/hyperactivity disorder, deve-
lopmental or intellectual disability).
Physical, mental or other inability to participate in treatment or as-
sessments (eg, impediment in hearing, vision or language).
Previous or planned bariatric surgery.
IFB, Integrated Research and Treatment Centre AdiposityDiseases.
Use of medication that impacts weight or executive functioning (eg,
antipsychotics, sedatives, hypnotics).
Current psychotherapy regarding weight or eating behaviour.
Current participation in other interventional studies.
Lack of compliance.
Pregnancy or lactation.

$50 \%$ chance of receiving CRT at no cost and financial incentives for participation in assessments (€80 total maximum, plus $€ 40$ maximum travel allowance) and treatment (€80 maximum travel allowance).

BWL treatment at the IFB Outpatient Unit requires BMI $\geq 35.0 \mathrm{~kg} / \mathrm{m}^{2}$ for admission. All patients receive BWL treatment at the IFB, consisting of an individual plus group-based lifestyle intervention on diet, physical activity and psychosocial aspects for up to 1 year. The intensity of BWL is tailored to an individual patient's needs and resources (eg, health insurance coverage).

\section{Procedures}

After enrolment for BWL treatment at the IFB Outpatient Unit, patients undergo a telephone screening (-t1), determining eligibility. Eligible patients are invited to the pre-treatment assessment ( $\mathrm{t} 0$ ), during which eligibility is confirmed, written informed consent is obtained, and patients are enrolled and centrally randomised to the CRT or control arm. (A sample copy of the consent form is available on request.) After 2 months of CRT versus no treatment, a post-treatment assessment $(\mathrm{t} 1)$ is conducted, and after 8 and 14 months following randomisation, 6-month and 12-month follow-up assessments (t2, t3) take place. The t 2 and $\mathrm{t} 3$ assessments correspond to mid-assessments and post-assessments in BWL at 6 and 12 months after start of this treatment. The CRT assessments include anthropometrics, executive functions (at $\mathrm{t} 0$ and $\mathrm{t} 1$ only), behavioural indicators related to weight loss, mental and physical health, and attendance of BWL treatment.

\section{Interventions}

\section{Experimental intervention: CRT}

The CRT manualised programme for obesity ${ }^{59}$ was broadened to address the full range of executive functioning deficits in individuals with obesity ${ }^{17} 19$ and adapted to pre-BWL use and group setting. The treatment focuses on mental exercises aimed at improving executive functions, including goal-setting, switching attention, inhibition and automatic behaviour, decision-making, planning, problem-solving, and flexibility through practice. A positive group climate is fostered, all patients are equally encouraged to engage in group sessions, and mental exercises including homework are individualised. Therapeutic sessions and topics are depicted in table 1.

CRT promotes reflection on thinking styles, develops metacognition and helps to explore and apply new thinking strategies in everyday life. An example exercise on flexibility, the Stroop task, asks participants to practice switching between different aspects of the stimuli or between different rules of the task quickly and accurately. CRT does not directly focus on weight loss, but exercises are tied in to this domain. CRT uses paper-and-pencil and computerised neuropsychological exercises (eg, on selective attention, planning and inhibition).$^{65}$

CRT is delivered in 8 weekly group sessions $(120 \mathrm{~min}$ each) with 6-10 patients over 8 weeks as a preparatory adjunct to BWL. Homework is given after every session and discussed in the following session. If a patient is not able to participate in a CRT session, the therapist writes him/her a letter with information about the session and sends material and homework assignments. Treatment fidelity is ensured through regular supervision by $\mathrm{AH}$, also preventing drift in treatment delivery. In order to support supervision, each session is documented by the therapist using notes and audio tapes.

\section{Control intervention: no treatment}

The control group does not receive treatment before entering BWL. As CRT for pre-BWL use has not been evaluated, no treatment is an adequate control condition for evaluating the existence of an effect and determining absolute effect size, ${ }^{66}$ particularly in psychological treatment trials. Usually, patients are not offered any treatment between enrolment at the IFB and start of BWL. Thus, the no treatment control condition corresponds to a treatment as usual control condition.

\section{Measures}

Primary outcome is the per cent weight change at 6-month follow-up (t2) compared with pre-treatment ( $\mathrm{t} 0)$, both derived from objectively measured body weight.

Secondary outcomes include per cent weight change from pre-treatment at 2 months (t1, post-treatment) and at 12-month follow-up (t3), derived from objectively measured body weight, in order to inform about treatment effects on early weight loss and on weight loss maintenance. Further, secondary outcomes consist of: executive functioning ( $\mathrm{t} 0, \mathrm{t} 1)$, as assessed through 
Table 1 Therapeutic sessions and topics in cognitive remediation therapy

\begin{tabular}{|c|c|}
\hline Sessions & Content \\
\hline (1) Introduction & $\begin{array}{l}\text { Getting to know each other } \\
\text { Introduction to cognitive remediation therapy }\end{array}$ \\
\hline (4) Automatisms and inhibition & $\begin{array}{l}\text { Discussion of homework } \\
\text { Introduction to automatic behaviour and inhibition } \\
\text { General tasks regarding automatisms and inhibition } \\
\text { - Weight management-related tasks regarding automatisms and inhibition }\end{array}$ \\
\hline (6) Planning & $\begin{array}{l}\text { Discussion of homework } \\
\text { Introduction to planning } \\
\text { General tasks regarding planning } \\
\text { Weight management-related tasks regarding planning }\end{array}$ \\
\hline (7) Problem-solving & $\begin{array}{l}\text { Discussion of homework } \\
\text { Introduction to problem-solving } \\
\text { General tasks regarding problem-solving } \\
\text { Weight management-related tasks regarding problem-solving }\end{array}$ \\
\hline (8) Conclusion & $\begin{array}{l}\text { Discussion of homework } \\
\text { Conclusion } \\
\text { Feedback } \\
\text { Farewell }\end{array}$ \\
\hline
\end{tabular}

the Iowa Gambling Task, ${ }^{67}$ Inhibition (Go/NoGo, StopSignal) ${ }^{68}$ Delay Discounting Task, ${ }^{69}$ Tower of London, ${ }^{70}$ Wisconsin Card Sorting Test, ${ }^{71}$ Trail Making Test ${ }^{72}$; weight loss-related behaviours ( $\mathrm{t} 0-\mathrm{t} 3$ ), including, self-efficacy (Generalised Self-Efficacy Scale), ${ }^{73}$ eating behaviour (Dutch Eating Behaviour Questionnaire) ${ }^{75} 76$ and physical activity (International Physical Activity Questionnaire) ${ }^{77}$; attendance to BWL sessions and retention as measures of patients' adherence to a weight loss regimen; mental health (t0-t3), operationalised as eating disorder psychopathology (Eating Disorder Examination-Questionnaire 8), ${ }^{78}$ general psychopathology (Patient Health Questionnaire) ${ }^{7980}$ and quality of life (Impact of Weight on Quality of Life $)^{81}{ }^{82}$; and physical health (t0-t3), for which hip and waist circumference, blood pressure, bioelectrical impedance, and triceps and subscapularis skinfolds are measured. We chose these outcome measures because they cover clinically relevant aspects, exhibit good psychometric properties and are well-established in the German language as well as commonly used in international research studies on obesity. Regarding repeated neuropsychological testing, it needs to be noted that in order to avoid potential learning effects, these tests are conducted at $\mathrm{t} 0$ and $\mathrm{t} 1$ only and that the no treatment control condition will control for these effects through retesting. ${ }^{83}$

As potential covariates, sociodemographic variables (t0-t3), measures of intelligence (Raven Matrices), ${ }^{84}$ working memory (N-Back), ${ }^{85}$ alertness ${ }^{86}$ and food addiction (Yale Food Addiction Scale 2.0) ${ }^{87} 88$ (t0) are assessed. Predictor variables are assessed at $\mathrm{t} 0$ and $\mathrm{t} 1$ and include all outcome variables, sociodemographic variables, weight history, expectations and motivation (rated on an 11-point Likert scale from 0 (not at all) to 10 (completely)) and compliance with CRT. In addition, patient evaluation of CRT is assessed at t1 (0 (not at all) to 10 (completely)).

All assessments are conducted by trained assessors, blinded to randomisation. They have undergone extensive training and receive ongoing supervision for drift prevention.

Every effort is made to retain as many patients as possible throughout the period of the study, including informing participants about the relevance and necessity of the study, use of continuity forms for locating participants, and use of incentives for post-treatment and follow-up assessments. 


\section{Methodological aspects}

\section{Power analysis}

The BWL programme at the IFB Outpatient Unit has been assessed for 200 patients and suggests that a weight loss of about $5 \% \pm 5.5 \%$ points can be expected for the control group completers with a $30 \%$ drop-out rate. We expect the completers in CRT to lose $2 \mathrm{~kg}$ more on average and have half the drop-out rate. Assuming no weight change for drop-out patients, but with a comparable variance to the completers, this translates to a weight loss of $7 \% \pm 5.5 \%$ points. This difference can be detected using a t-test with a power of $80 \%$ at a significance level of $5 \%$ by analysing 120 patients per arm. The cluster effect of the group sessions was modelled with an intraclass correlation coefficient for BMI at the postcode level of $0.004,{ }^{89}$ which would require only about one more group per arm to be enrolled. We, thus, plan on randomising 130 patients per arm, which already takes into account a drop-out rate of about $22 \%$ overall. This number of patients will allow for the consideration of various covariates in further exploratory analyses.

\section{Randomisation}

Patients meeting study criteria are randomised after giving written informed consent. Randomisation is performed electronically by a tool developed by the Clinical Trial Centre of the University of Leipzig to ensure allocation concealment. Randomisation is stratified by sex and age (above the age of 45 or not) and uses block of variable length. The allocation ratio between the two study arms is $1: 1$.

\section{Blinding}

Assessments are performed by independent, blinded assessors who have no therapeutic relationship with the patients. Blinding of patients is not possible because patients know the treatment conditions based on the specific modes of delivery. Blinding of therapists is not possible because of the no treatment control condition.

\section{Data analytical plan}

The primary endpoint, per cent weight change at 6-month follow-up, will be estimated for each arm along with $95 \%$ CI based on a normal approximation for the mean change. A mixed model with the stratification variables, randomisation arm and baseline weight as fixed effects and the therapy group as a random effect will be used to determine the efficacy of CRT compared with no treatment. Similar analyses will be performed for the 12-month follow-up. This confirmatory analysis will follow the intent-to-treat principle and will be based on the full analysis set. Every attempt will be made to acquire missing data. If data missing for the primary endpoint can be expected to bias results in a meaningful way, then multiple imputation will be performed assuming that drop-out do not have weight loss on average. Further, the analysis of the primary endpoint will be performed in the per-protocol set to evaluate the treatment effect for patients with good protocol adherence.
Secondary endpoints will be analysed in similar mixed model analyses and further covariates will be included to identify predictors of treatment outcome. In particular, maintenance of treatment success over time will be evaluated. Effect sizes will be presented along with 95\% CIs.

For safety analysis, adverse events and severe adverse events will be analysed descriptively. No interim analyses are planned. The study will be reported according to the Consolidated Standards of Reporting Trials criteria. ${ }^{90}$

\section{Monitoring and data management}

The trial is performed in cooperation with the Clinical Trial Centre of the University of Leipzig, which is responsible for monitoring and data management. Monitoring is based on the trial protocol and the standard operating procedures of the Clinical Trial Centre using a risk-based approach. It consists of a combination of on-site and central monitoring and is independent of the study team and the principal investigator. Details are specified in a monitoring manual.

Data capture is paper-based at the trial site. Data are entered into the trial database at the Clinical Trial Centre. Overnight routines systematically check data for completeness, consistency and plausibility by routines implemented in the clinical data management system (eRT by OmniCom), running every night. Error messages (in case of discrepancies, errors or omissions) generated by these routines are checked by data management staff of the Clinical Trial Centre and passed on to the principal investigator or an authorised member of the study team at the trial site for solution.

During and after trial implementation, data are collected and stored on servers of the Clinical Trial Centre, and thus behind the firewall of the University of Leipzig. Access to the servers is secured via https protocol, and requires user-specific login and password.

Post-treatment data will be released only after study completion (ie, after termination of the 12-month follow-up). AH will be granted access to the final trial dataset.

\section{Confidentiality}

All clinical data recorded at the trial site on paper case report forms will be entered into the database at the Clinical Trial Centre by using a trial identification number that does not reference the patient's personal identifiers (anonymised data). In the event of withdrawal of consent, data will be deleted as requested, with full documentation of the reasons for deletion. Data analysis will be performed solely using de-identified data. After trial publication, trial data will be shared in de-identified form on justified request.

Personal information about potential and enrolled participants collected during enrolment will only be stored at the trial site and be subject to the assessors' and therapists' privacy obligation. Personal information will not be shared and will be deleted after the trial. 


\section{Ethics and dissemination}

The study was approved by the Ethical Committee of the University of Leipzig (256-15-13072015). Written informed consent is obtained by trained staff after the study has been fully explained and prior to randomisation (a model consent form is available on request). Patients can withdraw at any time without any disadvantage. The trial is conducted in accordance to the guidelines for Good Clinical Practice (GCP) ${ }^{91}$ All persons participating in the conduct of the trial commit themselves to the Declaration of Helsinki, ${ }^{92}$ as well as all pertinent national laws and the International Council for Harmonisation of Technical Requirements for Pharmaceuticals for Human Use (ICH) guidelines for GCP and CPMP/ICH/135/95. ${ }^{66}$ For protocol modifications including changes to eligibility criteria, outcomes or analyses approval by the Ethical Committee of the University of Leipzig would be sought.

\section{Safety}

Adverse events are all unwanted medical events (eg, emerging or aggravating symptoms) occurring throughout the trial, whether or not they have a causal association with the trial. Adverse events will be recorded through a self-report assessment of somatic symptoms at every assessment. ${ }^{79}$ Serious adverse events are those that led to death, are life threatening, make inpatient treatment necessary, lead to sustained harm or cause birth defects or deformities. Serious adverse events include mental or physical decompensations that indicate a need for hospitalisation (eg, acute suicidality). Serious adverse events are documented in a case report form. Trial personnel will record the date of occurrence, duration and severity of the serious adverse event, along with treatments and consequences. Any serious adverse event is immediately reported to the principal investigator and the Clinical Trial Centre and entered into the trial database. Should central monitoring hint at an unexpected accumulation of serious adverse events overall or in one treatment arm, a root-cause analysis will be performed to enable corrective and protective action, for example, by adjustment of selection criteria, adding safety measurements or additional visits.

An independent data monitoring and safety committee was not deemed to be necessary, because patient safety is not affected by the intervention that focuses on executive functioning but not on psychopathology. In case of adverse events making ancillary or post-trial care necessary, patients are referred to psychological or medical treatment at the IFB Outpatient Unit or other local healthcare facilities. Since serious adverse events have never been reported in comparable treatment trials, ${ }^{5393-95}$ no provisions for compensation to those who might suffer harm from trial participation are made (eg, no patient insurance).

\section{Discontinuation}

A patient may withdraw from the study at any time, at his or her own request, for any reason, specified or unspecified, and without penalty or loss of benefits to which the patient is otherwise entitled.

Furthermore, the responsible investigator has the right to discontinue the treatment according to a set of stopping rules (eg, adverse or serious adverse events, unacceptable benefit:risk ratio, lack of compliance). If possible, date and reasons for discontinuation are documented in the

\begin{tabular}{lll}
\hline Table 2 Roles and responsibilities & \\
\hline & $\begin{array}{l}\text { Principal } \\
\text { investigator } \\
\text { and study } \\
\text { team }\end{array}$ & $\begin{array}{l}\text { Clinical Trial } \\
\text { Centre }\end{array}$ \\
\hline $\begin{array}{ll}\text { Study preparation } \\
\text { Study grant }\end{array}$ & $\mathrm{X}^{\star}$ & $\mathrm{X}$ \\
$\begin{array}{l}\text { Study protocol } \\
\text { Ethical approval and } \\
\text { amendments }\end{array}$ & $\mathrm{X}$ & $\mathrm{X}$ \\
Case report forms & $\mathrm{X}$ & $\mathrm{X}$ \\
Manualisation & $\mathrm{X}$ & $\mathrm{X}$ \\
Training and supervision & $\mathrm{X}$ & \\
\hline
\end{tabular}

Study conduct

\begin{tabular}{|c|c|c|}
\hline Recruitment & $X$ & \\
\hline Informed consent & $x$ & \\
\hline Randomisation & & $\mathrm{X}$ \\
\hline Allocation & $X$ & \\
\hline Treatment & $\mathrm{X}$ & \\
\hline Assessments & $X$ & \\
\hline Documentation & $\mathrm{X}$ & \\
\hline \multicolumn{3}{|l|}{ Data management } \\
\hline Database development & & $\mathrm{X}$ \\
\hline Data entry & & $x$ \\
\hline Data check & & X \\
\hline Data archiving & & $x$ \\
\hline Query management & $\mathrm{X}$ & $\mathrm{X}$ \\
\hline \multicolumn{3}{|l|}{ Quality management } \\
\hline Trial supervision & $X^{*}$ & \\
\hline $\begin{array}{l}\text { Assessment and reporting of } \\
\text { serious adverse events }\end{array}$ & $x$ & $x$ \\
\hline Risk/benefit evaluation & $x$ & $x$ \\
\hline Monitoring & & $\mathrm{X}$ \\
\hline \multicolumn{3}{|l|}{ Data analysis and reporting } \\
\hline Data analysis plan & & $x+$ \\
\hline Power analysis & & $x+$ \\
\hline Randomisation & & $x+$ \\
\hline Recruitment documentation & & $x \dagger$ \\
\hline Data analysis & & $x \dagger$ \\
\hline Reporting & $\mathrm{X}$ & $x \dagger$ \\
\hline
\end{tabular}

*Principal investigator (AH).

†Biometrician (DP). 
case report forms and a final examination is done. In any case, all patients will be followed up at each assessment time point after discontinuation according to the intention-to-treat principle.

Furthermore, the responsible investigator has the right to discontinue the trial, if information emerges that affects the benefit:risk ratio of the trial, actual recruitment and follow-up rates do not guarantee the necessary statistical power, or repeated serious adverse events presumably associated with the trial are observed.

\section{Dissemination}

The study results will be disseminated through peer-reviewed publications and conference presentations to the scientific community, and through further presentations to the public and healthcare professionals. No restrictions on publication exist. Authorship will follow the Rules of Good Scientific Practice of the German Research Foundation, ${ }^{96}$ and no professional writers will be used.

\section{Patient and public involvement}

The development of the research question was informed by our clinical experience with patients' difficulties in BWL treatment, likely to result from deficits in executive functioning. However, patients were not involved in planning or conduct of the CRT study. Patient evaluation of CRT is measured. Patients will be informed about the study results through postings at the Outpatient Unit of the IFB AdiposityDiseases and its website as well as through presentations to the public.

\section{Roles and responsibilities}

The roles and responsibilities within the CRT study are summarised in table 2. An independent data monitoring and safety committee was not involved (see the Safety section).

\section{Author affiliations}

${ }^{1}$ Integrated Research and Treatment Centre AdiposityDiseases, Department of Medical Psychology and Medical Sociology, Department of Psychosomatic Medicine and Psychotherapy, University of Leipzig Medical Center, Leipzig, Germany ${ }^{2}$ Clinical Trial Centre Leipzig, University of Leipzig, Leipzig, Germany ${ }^{3}$ School of Social Sciences and Psychology, Western Sydney University, Sydney, New South Wales, Australia

${ }^{4}$ Translational Health Research Institute, Western Sydney University, Sydney, New South Wales, Australia

Acknowledgements We are grateful to Jamie L Manwaring and to Frederike Obereigner for their help in editing this paper.

Contributors $\mathrm{AH}$ (principal investigator) conceived and designed the study with input from DP (biometrician), PN, ES, PJH and MB. AH, MB, CH, DP and PN wrote the study protocol with contribution from ES, PJH and MB.

Funding This work was supported by the grant (01E01501) from the German Federal Ministry of Education and Research.

Disclaimer The funding body has no influence on the study design, data collection, writing of the manuscript or in the decision to submit the manuscript for publication.

Competing interests None declared.

Patient consent Not required.

Ethics approval The study was approved by the Ethical Committee of the University of Leipzig (256-15-13072015).
Provenance and peer review Not commissioned; externally peer reviewed.

Open access This is an open access article distributed in accordance with the Creative Commons Attribution Non Commercial (CC BY-NC 4.0) license, which permits others to distribute, remix, adapt, build upon this work non-commercially, and license their derivative works on different terms, provided the original work is properly cited, appropriate credit is given, any changes made indicated, and the use is non-commercial. See: http://creativecommons.org/licenses/by-nc/4.0/.

\section{REFERENCES}

1. NCD Risk Factor Collaboration (NCD-RisC). Worldwide trends in body-mass index, underweight, overweight, and obesity from 1975 to 2016: a pooled analysis of 2416 population-based measurement studies in 128.9 million children, adolescents, and adults. Lancet 2017;390:2627-42.

2. $\mathrm{Ng} \mathrm{M}$, Fleming $\mathrm{T}$, Robinson $\mathrm{M}$, et al. Global, regional, and national prevalence of overweight and obesity in children and adults during 1980-2013: a systematic analysis for the Global Burden of Disease Study 2013. Lancet 2014;384:766-81.

3. Afshin A, Forouzanfar MH, Reitsma MB, et al. Health effects of overweight and obesity in 195 countries over 25 years. N Engl J Med 2017;377:13-27.

4. Global BMI Mortality Collaboration, Di Angelantonio E, Bhupathiraju $\mathrm{S}$, et al. Body-mass index and all-cause mortality: individualparticipant-data meta-analysis of 239 prospective studies in four continents. Lancet 2016;388:776-86.

5. Ma C, Avenell A, Bolland M, et al. Effects of weight loss interventions for adults who are obese on mortality, cardiovascular disease, and cancer: systematic review and meta-analysis. BMJ 2017;359:j4849.

6. Zomer E, Gurusamy K, Leach R, et al. Interventions that cause weight loss and the impact on cardiovascular risk factors: a systematic review and meta-analysis. Obes Rev 2016;17:1001-11.

7. Franz MJ, Boucher JL, Rutten-Ramos S, et al. Lifestyle weight-loss intervention outcomes in overweight and obese adults with type 2 diabetes: a systematic review and meta-analysis of randomized clinical trials. J Acad Nutr Diet 2015;115:1447-63.

8. Peirson L, Douketis J, Ciliska D, et al. Treatment for overweight and obesity in adult populations: a systematic review and meta-analysis. CMAJ Open 2014;2:E306-17.

9. Ryan $\mathrm{DH}$, Kahan S. Guideline recommendations for obesity management. Med Clin North Am 2018;102:49-63.

10. Peirson L, Fitzpatrick-Lewis D, Ciliska D, et al. Strategies for weight maintenance in adult populations treated for overweight and obesity: a systematic review and meta-analysis. CMAJ Open 2015;3:E47-54.

11. Shallice T. From neuropsychology to mental structure: Cambridge University Press, 1988.

12. Stuss DT, Benson DF. The frontal lobes: Raven Press, 1986.

13. Hofmann W, Schmeichel BJ, Baddeley AD. Executive functions and self-regulation. Trends Cogn Sci 2012;16:174-80.

14. Fitzpatrick S, Gilbert S, Serpell L. Systematic review: are overweight and obese individuals impaired on behavioural tasks of executive functioning? Neuropsychol Rev 2013;23:138-56.

15. Smith $\mathrm{E}$, Hay $\mathrm{P}$, Campbell $\mathrm{L}$, et al. A review of the association between obesity and cognitive function across the lifespan: implications for novel approaches to prevention and treatment. Obes Rev 2011;12:740-55.

16. Kittel R, Brauhardt A, Hilbert A. Cognitive and emotional functioning in binge-eating disorder: a systematic review. Int J Eat Disord 2015;48:535-54.

17. Yang Y, Shields GS, Guo C, et al. Executive function performance in obesity and overweight individuals: a meta-analysis and review. Neurosci Biobehav Rev 2018;84:225-44.

18. Martin AA, Davidson TL. Human cognitive function and the obesogenic environment. Physiol Behav 2014;136:185-93.

19. Gettens KM, Gorin AA. Executive function in weight loss and weight loss maintenance: a conceptual review and novel neuropsychological model of weight control. J Behav Med 2017;40:687-701.

20. Dohle S, Diel K, Hofmann W. Executive functions and the selfregulation of eating behavior: a review. Appetite 2018;124:30160-5.

21. Raman J, Smith E, Hay P. The clinical obesity maintenance model: an integration of psychological constructs including mood, emotional regulation, disordered overeating, habitual cluster behaviours, health literacy and cognitive function. J Obes 2013;2013:1-9.

22. Elfhag K, Rössner $\mathrm{S}$. Who succeeds in maintaining weight loss? A conceptual review of factors associated with weight loss maintenance and weight regain. Obes Rev 2005;6:67-85.

23. Stubbs J, Whybrow S, Teixeira $P$, et al. Problems in identifying predictors and correlates of weight loss and maintenance: 
implications for weight control therapies based on behaviour change. Obes Rev 2011;12:688-708.

24. Wyckoff EP, Evans BC, Manasse SM, et al. Executive functioning and dietary intake: neurocognitive correlates of fruit, vegetable, and saturated fat intake in adults with obesity. Appetite 2017;111:79-85.

25. Allan JL, Johnston M, Campbell N. Unintentional eating. What determines goal-incongruent chocolate consumption? Appetite 2010;54:422-5

26. Allom V, Mullan B. Individual differences in executive function predict distinct eating behaviours. Appetite 2014;80:123-30.

27. Houben K. Overcoming the urge to splurge: influencing eating behavior by manipulating inhibitory control. J Behav Ther Exp Psychiatry 2011;42:384-8.

28. Hall PA, Fong GT, Epp LJ, et al. Executive function moderates the intention-behavior link for physical activity and dietary behavior. Psychol Health 2008;23:309-26.

29. Nederkoorn C, Houben K, Hofmann W, et al. Control yourself or just eat what you like? Weight gain over a year is predicted by an interactive effect of response inhibition and implicit preference for snack foods. Health Psychol 2010;29:389-93.

30. Stinson EJ, Krakoff J, Gluck ME. Depressive symptoms and poorer performance on the Stroop Task are associated with weight gain. Physiol Behav 2018;186:25-30.

31. Witbracht MG, Laugero KD, Van Loan MD, et al. Performance on the lowa Gambling Task is related to magnitude of weight loss and salivary cortisol in a diet-induced weight loss intervention in overweight women. Physiol Behav 2012;106:291-7.

32. Handley JD, Williams DM, Caplin S, et al. Changes in cognitive function following bariatric surgery: a systematic review. Obes Surg 2016;26:2530-7.

33. Veronese N, Facchini S, Stubbs B, et al. Weight loss is associated with improvements in cognitive function among overweight and obese people: a systematic review and meta-analysis. Neurosci Biobehav Rev 2017;72:87-94.

34. Siervo M, Arnold R, Wells JC, et al. Intentional weight loss in overweight and obese individuals and cognitive function: a systematic review and meta-analysis. Obes Rev 2011;12:968-83.

35. Alfaro FJ, Gavrieli A, Saade-Lemus P, et al. White matter microstructure and cognitive decline in metabolic syndrome: a review of diffusion tensor imaging. Metabolism 2018;78:52-68.

36. Alosco ML, Gunstad J. The negative effects of obesity and poor glycemic control on cognitive function: a proposed model for possible mechanisms. Curr Diab Rep 2014;14:495.

37. Lasselin J, Magne E, Beau C, et al. Low-grade inflammation is a major contributor of impaired attentional set shifting in obese subjects. Brain Behav Immun 2016;58:63-8.

38. Guillemot-Legris O, Muccioli GG. Obesity-induced neuroinflammation: beyond the hypothalamus. Trends Neurosci 2017;40:237-53.

39. Miller AA, Spencer SJ. Obesity and neuroinflammation: a pathway to cognitive impairment. Brain Behav Immun 2014;42:10-21.

40. Marioni RE, Yang J, Dykiert D, et al. Assessing the genetic overlap between BMI and cognitive function. Mol Psychiatry 2016;21:1477-82.

41. Stice E, Burger KS, Yokum S. Reward region responsivity predicts future weight gain and moderating effects of the TaqIA Allele. $J$ Neurosci 2015;35:10316-24.

42. García-García I, Narberhaus A, Marqués-Iturria I, et al. Neural responses to visual food cues: insights from functional magnetic resonance imaging. Eur Eat Disord Rev 2013;21:89-98.

43. Chen J, Papies EK, Barsalou LW. A core eating network and its modulations underlie diverse eating phenomena. Brain Cogn 2016;110:20-42.

44. Weygandt M, Mai K, Dommes E, et al. Impulse control in the dorsolateral prefrontal cortex counteracts post-diet weight regain in obesity. Neuroimage 2015;109:318-27.

45. Weygandt M, Mai K, Dommes E, et al. The role of neural impulse control mechanisms for dietary success in obesity. Neuroimage 2013:83:669-78.

46. Jones A, Hardman CA, Lawrence N, et al. Cognitive training as a potential treatment for overweight and obesity: A critical review of the evidence. Appetite 2018;124:50-67.

47. Luria AR. The man with a shattered world: the history of a brain wound: Harvard University Press, 1972

48. Harvey PD, Bowie CR. Cognitive enhancement in schizophrenia: pharmacological and cognitive remediation approaches. Psychiatr Clin North Am 2012;35:683-98.

49. Bowie CR, Gupta M, Holshausen K. Cognitive remediation therapy for mood disorders: rationale, early evidence, and future directions. Can J Psychiatry 2013;58:319-25.
50. Stevenson CS, Whitmont S, Bornholt L, et al. A cognitive remediation programme for adults with Attention Deficit Hyperactivity Disorder. Aust N Z J Psychiatry 2002;36:610-6.

51. Lindvall Dahlgren $C, R ø O$. A systematic review of cognitive remediation therapy for anorexia nervosa - development, current state and implications for future research and clinical practice. $J$ Eat Disord 2014;2:26.

52. Tchanturia K, Giombini L, Leppanen J, et al. Evidence for cognitive remediation therapy in young people with anorexia nervosa: systematic review and meta-analysis of the literature. Eur Eat Disord Rev 2017;25:227-36.

53. Raman J, Hay P, Tchanturia K, et al. A randomised controlled trial of manualized cognitive remediation therapy in adult obesity. Appetite 2018;123:269-79.

54. Smith E, Whittingham C. Cognitive remediation therapy plus behavioural weight loss compared to behavioural weight loss alone for obesity: study protocol for a randomised controlled trial. Trials 2017:18:42.

55. Teixeira PJ, Silva MN, Mata J, et al. Motivation, self-determination, and long-term weight control. Int J Behav Nutr Phys Act 2012;9:22.

56. Armstrong MJ, Mottershead TA, Ronksley PE, et al. Motivational interviewing to improve weight loss in overweight and/or obese patients: a systematic review and meta-analysis of randomized controlled trials. Obes Rev 2011;12:709-23.

57. Specchia ML, Veneziano MA, Cadeddu C, et al. Economic impact of adult obesity on health systems: a systematic review. Eur J Public Health 2015;25:255-62.

58. Tremmel M, Gerdtham UG, Nilsson PM, et al. Economic burden of obesity: a systematic literature review. Int J Environ Res Public Health 2017;14:435.

59. Smith E, Hay P, Raman J. Cognitive remediation therapy adaptation for obesity. Tchanturia K, ed. Cognitive Remediation Therapy (CRT) for eating and weight disorders. London: Routledge, 2014.

60. Field M, Werthmann J, Franken I, et al. The role of attentional bias in obesity and addiction. Health Psychol 2016;35:767-80.

61. Enriquez-Geppert S, Huster RJ, Herrmann CS. Boosting brain functions: improving executive functions with behavioral training, neurostimulation, and neurofeedback. Int J Psychophysiol 2013;88:1-16.

62. Forman EM, Goldstein SP, Flack D, et al. Promising technological innovations in cognitive training to treat eating-related behavior. Appetite 2018;124.

63. Deutsche Adipositas-Gesellschaft (DAG) e.V. Interdisziplinäre Leitlinie der Qualität S3 zur „Prävention und Therapie der Adipositas". 2014 http://www.adipositas-gesellschaft.de/fileadmin/PDF/Leitlinien/050001I S3 Adipositas_Praevention Therapie_2014-11.pdf (accessed 23 May 2018).

64. World Health Organisation (WHO). Obesity and overweight: Fact sheet. 2017 http://www.who.int/mediacentre/factsheets/fs311/en/ (accessed 30 Jul 2017).

65. Dieckhut C, Lünsdorf O, Enriquez-Geppert S. Kognitives Training. Oldenburg: Carl von Ossietzky Universität, 2014.

66. International Conference on Harmonisation of technical requirements for registration of pharmaceuticals for human use. ICH harmonized tripartite guideline: guideline for good clinical practice. J Postgrad Med 2001;47:45-50.

67. Bechara A, Damasio AR, Damasio $\mathrm{H}$, et al. Insensitivity to future consequences following damage to human prefrontal cortex. Cognition 1994;50:7-15.

68. Kaiser S, Aschenbrenner S, Pfüller U, et al. Wiener Testsystem: Response Inhibition. Mödling: Schuhfried, 2015.

69. Richards JB, Zhang L, Mitchell SH, et al. Delay or probability discounting in a model of impulsive behavior: effect of alcohol. $J$ Exp Anal Behav 1999;71:121-43.

70. Kaller CP, Unterrainer JM, Kaiser S, et al. Wiener Testsystem: Tower of London - Freiburger Version. Mödling: Schuhfried, 2015.

71. Heaton RK, Chelune GJ, Talley JL, et al. Wisconsin card sorting test manual. Odessa, Florida: Psychological Assessment Resources, Inc, 1993.

72. Rodewald K, Weisbrod M, Aschenbrenner S. Wiener Testsystem: Trail Making Test - Langensteinbacher Version. Mödling: Schuhfried, 2015.

73. Schwarzer R, Jerusalem M. Generalized self-efficacy scale. In: Weinman J, Wright S, Johnston M, eds. Measures in health psychology: a user's portfolio. Causal and control beliefs. Windsor, England: Nfer-nelson, 1995:35-7.

74. Schwarzer R, Jerusalem M. Skalen zur Erfassung von Lehrer- und Schülermerkmalen. In: Dokumentation der psychometrischen Verfahren im Rahmen der Wissenschaftlichen Begleitung des Modellversuchs Selbstwirksame Schulen. Berlin: Freie Universität Berlin, 1999. 
75. van Strien T, Frijters JER, Bergers GPA, et al. The Dutch Eating Behavior Questionnaire (DEBQ) for assessment of restrained, emotional, and external eating behavior. Int J Eat Disord 1986:5:295-315.

76. Pudel V, Westenhöfer J. Fragebogen zum Essverhalten. Göttingen: Hogrefe, 1989

77. Craig CL, Marshall AL, Sjöström M, et al. International physical activity questionnaire: 12 -country reliability and validity. Med Sci Sports Exerc 2003;35:1381-95.

78. Kliem S, Mößle T, Zenger M, et al. The Eating Disorder ExaminationQuestionnaire 8: a brief measure of eating disorder psychopathology (EDE-Q8). Int J Eat Disord 2016;49:613-6.

79. Gräfe K, Zipfel S, Herzog W, et al. Screening psychischer Störungen mit dem "Gesundheitsfragebogen für Patienten (PHQ-D)". Diagnostica 2004;50:171-81.

80. Spitzer RL, Kroenke K, Williams JB. Validation and utility of a selfreport version of PRIME-MD: the PHQ primary care study. Primary Care Evaluation of Mental Disorders. Patient Health Questionnaire. JAMA 1999:282:1737-44.

81. Kolotkin RL, Crosby RD, Kosloski KD, et al. Development of a brief measure to assess quality of life in obesity. Obes Res 2001;9:102-11.

82. Mueller A, Holzapfel $\mathrm{C}$, Hauner $\mathrm{H}$, et al. Psychometric evaluation of the German version of the Impact of Weight on Quality of LifeLite (IWQOL-Lite) questionnaire. Exp Clin Endocrinol Diabetes 2011;119:69-74.

83. Paap KR, Sawi O. The role of test-retest reliability in measuring individual and group differences in executive functioning. J Neurosci Methods 2016;274:81-93.

84. Raven JC, Court JH, Raven J. Wiener Testsystem: Raven's Advanced Progressive Matrices. Mödling: Schuhfried, 2015.

85. Schellig D, Schuri U. Wiener Testsystem: N-Back Verbal. Mödling: Schuhfried, 2015.

86. Sturm W. Wiener Testsystem: Raven's Advanced Progressive Matrices. Mödling: Schuhfried, 2015.
87. Gearhardt AN, Corbin WR, Brownell KD. Development of the Yale Food Addiction Scale version 2.0. Psychol Addict Behav 2016;30:113-21.

88. Meule A, Müller A, Gearhardt AN, et al. German version of the Yale Food Addiction Scale 2.0: prevalence and correlates of 'food addiction' in students and obese individuals. Appetite 2017:115:54-61.

89. Ukoumunne OC, Gulliford MC, Chinn S, et al. Methods for evaluating area-wide and organisation-based interventions in health and health care: a systematic review. Health Technol Assess 1999;3:iii-92.

90. Moher D. CONSORT: an evolving tool to help improve the quality of reports of randomized controlled trials. Consolidated Standards of Reporting Trials. JAMA 1998;279:1489-91.

91. Bundesministerium der Justiz. Verordnung über die Anwendung der Guten Klinischen Praxis bei der Durchführung von klinischen Prüfungen mit Arzneimitteln zur Anwendung am Menschen (GCPVerordnung - GCP-V). 2012 https://www.gesetze-im-internet.de/gcpv/BJNR208100004.html (accessed 8 Feb 2018).

92. World Medical Association. Declaration of Helsinki: guiding physicians in biomedical research involving human subjects. 2013 https://www.wma.net/what-we-do/medical-ethics/declaration-ofhelsinki/ (accessed 8 Feb 2018).

93. Brockmeyer $\mathrm{T}$, Ingenerf $\mathrm{K}$, Walther $\mathrm{S}$, et al. Training cognitive flexibility in patients with anorexia nervosa: a pilot randomized controlled trial of cognitive remediation therapy. Int J Eat Disord 2014;47:24-31.

94. Lock J, Agras WS, Fitzpatrick KK, et al. Is outpatient cognitive remediation therapy feasible to use in randomized clinical trials for anorexia nervosa? Int J Eat Disord 2013;46:567-75.

95. Verbeken S, Braet C, Goossens L, et al. Executive function training with game elements for obese children: a novel treatment to enhance self-regulatory abilities for weight-control. Behav Res Ther 2013;51:290-9.

96. Deutsche Forschungsgemeinschaft. Sicherung Guter Wissenschaftlicher Praxis: Wiley-VCH Verlag GmbH \& Co. KGaA, 2013:1-109. 